\title{
EQUIDAD Y EFECTOS DISTRIBUTIVOS DE LA POLÍTICA REGIONAL DE INCLUSIÓN DIGITAL EN ANDALUCÍA
}

\author{
Gustavo Antonio Contreras Cabrera, Rosa María Jordá Borrell y \\ Javier López Otero \\ Departamento de Geografía Física y Análisis Geográfico Regional \\ Facultad de Geografía e Historia (Universidad de Sevilla)
}

\section{RESUMEN}

La corrección de la brecha digital en Andalucía ha sido investigada desde una doble perspectiva. En primer lugar, se ha llevado a cabo un reconocimiento de las principales interpretaciones de este nuevo fenómeno, contextualizándolo en el caso concreto de la política regional aplicada en la Comunidad Autónoma de Andalucía y explorado el origen e implantación de la estrategia Guadalinfo de alfabetización, acceso y uso y dinamización ciudadana basada en el despliegue de centros de acceso público a Internet y el manejo de las tecnologías de la información y la comunicación (TIC) por parte de su población objetivo.

Asimismo, se han examinado los impactos más destacados que la acción Guadalinfo ha tenido en dicha población, obteniendo interesantes implicaciones sobre la equidad e idoneidad de esta política regional y sus posibilidades de futuro.

Estos resultados mostrados son fruto de un proyecto desarrollado durante los años 2012 al 2014, tomando la gestión de la red de centros Guadalinfo extendida en el territorio andaluz como referente directo de dicha política y utilizando como fuente específica de información una encuesta propia realizada a los usuarios de dichos centros.

Palabras clave: brecha digital, política regional, equipamientos públicos, Andalucía

ABSTRACT

Equity and distributional effects of the regional policy of digital inclusion in Andalusia

The correction of the digital divide in Andalusia has been investigated from two perspectives. First, a reconnaissance of the main interpretations of this new phenomenon 
has been carried out, considering the case of the regional policy in Andalusia, and it has been explored the origin and implementation of the Guadalinfo strategy of literacy, as well as the access and use and citizen-based dynamic display of centers of public access to the Internet and the management of the information and communication technologies (ICT) by its target population.

It has also been considered the most important impacts that the Guadalinfo action has had in this population, obtaining some interesting results in terms of the fairness and adequacy of the regional policy and its future possibilities.

This paper shows the results of a research conducted between 2012 and 2014, taking the management of the Guadalinfo centers network, extended in the Andalusian territory, as a direct reference of the above mentioned policy and considering its users surveyed as a specific source of information.

Keywords: digital divide, regional policy, public facilities, Andalusia

\section{INTRODUCCIÓN}

En Andalucía (España) existen oportunidades y aptitudes ciudadanas diversas en el acceso y uso de las tecnologías de la información y la comunicación (TIC), así como de Internet. Para afrontar la brecha producida entre ciudadanos en base a las tecnologías y servicios digitales ha sido impulsada desde la Administración Autonómica la estrategia de centros de acceso, alfabetización y dinamización digital denominada Guadalinfo. Se trata de una política correctora de la inequidad regional gestionada desde el año 2002 en forma de red territorial de atención ciudadana, cuyos impactos más destacados han sido examinados y evaluados. Los resultados obtenidos evidencian carencias y posibles mejoras en su labor inclusiva en la sociedad de la información.

El examen de las políticas correctoras de la brecha digital en esta Comunidad Autónoma admite un extenso abanico de enfoques y tratamientos acerca de las implicaciones y capacidades de diseño de estrategias válidas para afrontar con mayor efectividad la integración ciudadana en la sociedad de la información, -de hecho, existen importantes estudios globales centrados en las capacidades de acceso y uso de las TIC e Internet por parte de los individuos/hogares o de las empresas, entre otras-. No obstante, se ha pretendido aquí abordar la cuestión desde una perspectiva más específica, centrada en determinados efectos producidos tras el despliegue por parte de la Administración Autonómica de Andalucía de su estrategia compensadora Guadalinfo, basada en la puesta en funcionamiento de un servicio público en red para el acceso, la alfabetización y la dinamización sociodigital, como los beneficios concretos aportados a la ciudadanía o el potencial de las prestaciones de sus centros de inclusión digital.

Con carácter previo, ha sido oportuno realizar un acercamiento al ideario político-institucional existente en torno a la brecha digital y a la propia equidad 
socioterritorial basada en las TIC, además de afrontar el proceso de configuración de la estrategia regional de inclusión digital fijada en Andalucía. Los aspectos y particularidades aquí mostrados constituyen una sólida base argumental para explicar la idoneidad de la red Guadalinfo desarrollada y una posterior interpretación de los impactos identificados, partiendo de la investigación efectuada durante los años 2012-2014', con la idea de comprobar la efectividad de la programación estratégica común de servicios Guadalinfo, considerando que la exclusión digital es inherente a la propia segregación social existente.

Las conclusiones expresadas al final del trabajo suponen el argumentario que sintetiza todo el proceso de investigación llevado a cabo, razonando en torno a los resultados obtenidos, señalando los principales avances producidos respecto del objeto del estudio y realizando algunas sugerencias sobre los aportes de esta investigación.

\section{IDEARIO POLÍTICO-INSTITUCIONAL SOBRE LA BRECHA DIGITAL}

Siguiendo las principales interpretaciones planteadas sobre la pobreza y la exclusión social, parece viable asimilar una situación de exclusión digital a la carencia de medios, de aptitudes o de oportunidades para la plena integración en la sociedad de la información, más transitoria que estructural, a la vez que se evidencia la posibilidad de acometer correcciones mediante políticas de lucha contra la brecha digital. En sintonía con esta idea, parece adecuado fijar como referente de base una definición oficialmente expresada sobre esta divisoria digital, empleando para ello la proporcionada por la Organización para la Cooperación y el Desarrollo Económico, que percibe la inequidad entre personas, familias, empresas e incluso áreas geográficas, en diferentes niveles socioeconómicos con respecto a sus posibilidades de acceso a las TIC y también para el empleo de Internet ante una diversidad considerable de actividades y aprovechamientos (OCDE, 2001).

Aun reconociendo la validez y aceptación de esa definición, es significativa la variedad de enfoques vinculados al imaginario político-institucional, de especial importancia reveladora por fijar los soportes ideológicos de su consecuente actuación contra la brecha. Así, determinados autores consideran ineludible la explicación de las políticas de inclusión digital de acuerdo con dicha multiplicidad de criterios que podrían coincidir con las apreciaciones que se muestran a continuación (Serrano y Martínez, 2003; Camacho, 2005).

En unos casos se interpreta una relación de causalidad directa entre el acceso a las TIC y las oportunidades de desarrollo, como expresión de un determinismo

1. Vinculada al Proyecto de evaluación del Plan Estratégico Guadalinfo y la definición estratégica para la creación e implantación de iniciativas de estímulo de los desafíos de su red en Andalucía (Instituto de Desarrollo Regional, Fundación de la Universidad de Sevilla). 
tecnológico. Estas oportunidades aparecen expresadas en forma de mejores condiciones de vida y bienestar, de reducción de la pobreza y de aprovechamiento de las potenciales ventajas tecnológicas para el desarrollo humano. En sentido contrario, un menor acceso a las TIC implica mayores dificultades para alcanzar ese desarrollo (García, 2005; Servon y Pinkett, 2006; Karakaya, 2012). Sin embargo, esta causalidad no es clara en la práctica, y los resultados que se producen tienden a considerarse como una solución sin explicación empírica, dada la propia complejidad de la brecha digital, su densa interconexión con el conjunto de las dinámicas sociales, su historia, su estructura organizativa, así como la intensidad y magnitud del proceso de incorporación de las TIC (Castells, 2006; Chaparro, 2007).

Ante el planteamiento causa-efecto en el desarrollo social puede considerarse la validez de su incidencia, pero como un componente del complejo crecimiento y perfeccionamiento de la sociedad, advirtiéndose un escenario de posibilismo tecnológico, con diversas oportunidades que tienden a hacer de la brecha un fenómeno circunstancial, contemplando la capacidad humana para transformar las TIC en una evolución innovadora dotada de identidad social (Downing, 2000; Mattelart, 2007).

Por otro lado, la idea de que los integrados en la sociedad de la información podrán desarrollarse plenamente y participar en el fortalecimiento de este nuevo modelo social ha calado también en el ideario político-institucional, -a modo de evolucionismo digital destinado a una nueva sociedad-, apreciándose con especial entusiasmo el papel dinamizador de las TIC. Este pensamiento otorga un lugar predominante a quienes sean capaces de alcanzar una buen uso y aprovechamiento de esas tecnologías, como grupo excepcional con capacidad innovadora, de influencia y decisión en el impulso y desarrollo de la sociedad de la información resultante (López y Sánchez, 2001).

En un contexto social donde el factor tecnológico es parte fundamental y generador de dependencia, la capacidad de acceso a las TIC y su aprovechamiento representan exigencias indiscutibles, apareciendo la divisoria digital como un síntoma de desequilibrio o distorsión (Comisión Europea, 2010). La idea de una brecha digital desencadenante de brechas sociales supone una variación del primer enfoque causa-efecto observado, reflexionándose en torno a dicha relación y a su capacidad de provocar cambios inmediatos. Allá donde existan diferencias para acceder y aprovechar las TIC se producirá un incremento en las diferencias sociales ya existentes (Brandtzaeg et al., 2014), e igual que se admiten diferentes variantes de brecha digital, se aprecia el carácter polisémico y la complejidad de la brecha social (DiMaggio et al. 2004).

Desde un punto de vista geográfico, la divisoria digital entre países, regiones o comunidades es asociada a mejores condiciones generales de acceso y conocimiento del manejo de las TIC, y al mismo tiempo se señala una inequidad 
interna en esos territorios, según las capacidades de acceso y uso TIC de su población, implicando la intensificación de grupos sociales dotados de mejores condiciones y oportunidades económicas, políticas, sociales o culturales. Este planteamiento hace pensar en enfoques o visiones del territorio y del desarrollo socioeconómico marcadas por conflictos de dependencia (Sampedro, 2013) o incluso de necesidades correctoras por parte de los poderes públicos atendiendo a una nueva generación de derechos globales para el desarrollo humano (López y Samek, 2009).

Existe también un discurso reduccionista de la brecha digital que se apoya en la carencia de medios TIC por parte de la población, muy extendido en el plano político-institucional por su fácil respuesta de inmersión sociodigital, -a modo de proceso acumulativo de aportes tecnológicos de la información, la comunicación e Internet-. Así, se tiende a considerar la brecha digital como un fenómeno desvinculado de otros aspectos sociales, económicos, culturales o ideológicos, afrontable mediante el incentivo o el aporte infotecnológico directo (Davenport, 1997). Este discurso soslaya sus múltiples características en el tiempo y el espacio social existentes, así como sus causas y consecuencias, pudiendo ofrecer soluciones en muchos casos difusas y carentes de una visión detallada del problema (Marco, 2000; Gutiérrez, 2007). La intención de recomponer una situación de partida asimétrica no llegaría a evitar nuevos desequilibrios, sin considerar las múltiples causas que la originan.

Para otros autores e instituciones (Manzanares, 2007; Guerra y Jordán, 2010) el esfuerzo corrector de carencias tecnológicas debe ser completado mejorando la capacitación ciudadana y la identificación de potencialidades que conlleva la universalización del conocimiento, aunque no siempre es formulada una misma estrategia para abordar el problema en los diferentes territorios, especialmente en la escala internacional, desde la visión de consenso para impulsar un plan de acción conjunta. Al contrario que la percepción oficial de la brecha como resultado único de la incapacidad de acceso digital, mencionada anteriormente, se concibe la idea de una sociedad de la información en desarrollo constante, que modela el uso y aprovechamiento de estas tecnologías y aplicativos con diversas intensidades y con usos variados, dependiendo de las necesidades, preferencias o potencialidades percibidas por la propia ciudadanía (Van Winden, 2003). Este ideario afronta una reconversión continua en su comprensión del fenómeno, analizándolo en su multidimensionalidad, entendiendo su complejidad y asumiendo la dificultad para impulsar medidas correctoras únicas (Johnston y Webber, 1999; Bawden, 2005).

Advertida la pluralidad de enfoques o interpretaciones sobre la brecha digital y sus posibles maneras de afrontarla desde los poderes públicos, cabe admitir una cuestión esencial que acompaña a todas las fórmulas de intervención. La excesiva preocupación por la reducción de la brecha digital llega a ocasionar 
reflexiones sesgadas de la realidad que conforma la sociedad de la información. Por consiguiente, el voluntarismo corrector de la inequidad digital llega a dificultar la búsqueda de resultados igualmente trascendentes, como asegurar unas mejores condiciones de bienestar ciudadano a través de las TIC, promover una mayor participación de la población en los procesos deliberativos sobre asuntos de interés general o profundizar en la prestación de unos servicios públicos de calidad, accesibles y eficaces (Norris, 2001).

Entrando en el campo concreto de la inclusión socioterritorial basada en las TIC, algunos autores (Van Dijk, 2005; Silver, 2009) resaltan la paradoja del papel de estas tecnologías como herramientas idóneas. Especialmente destacado es el planteamiento de inequidad de la sociedad de la información en el sentido de que las TIC son en si mismas causantes de la brecha en la sociedad que ayudan a construir. Así, la innovación tecnológica es asimilada con mayor intensidad y facilidad por las personas y grupos más conectados, de modo que las distancias entre éstos y las personas o grupos con menor acceso a las TIC aumentarán debido a las ventajas asociadas a la anticipación en su uso y aprovechamiento.

Por otra parte, la comprensión de la inclusión socioterritorial basada en las TIC parece tener una inevitable vinculación con los conceptos de cohesión social y cohesión territorial. Mientras que la exclusión social arroja sentido a su inverso conceptual, haciendo referencia a la capacidad de integración, de ruptura de las diferencias sociales más irreconciliables en el seno de la nueva sociedad de la información (Hernández, 2008), la cohesión territorial adquiere también un significado determinante en el contexto de la investigación de la acción estratégica de inmersión en la sociedad de la información.

Entre los planteamientos o enfoques reconocidos acerca de la forma de conseguir la cohesión territorial destaca el relativo a la prestación de servicios públicos de inclusión digital, tanto universales como personales. Estas tipologías tienen una impronta diferente en el territorio, aunque ambas contribuyen a su cohesión (Tarroja y Camagni, 2006). Los servicios universales son asumidos como fórmula de equidad, homogeneidad y estandarización en el territorio, -sujetos a un modelo de gestión centralizada y con la clara intención de garantizar su cobertura a todos los ciudadanos-, y los servicios personales responden más bien a necesidades coyunturales o problemas específicos que originan o surgen de una descompensación o heterogeneidad territorial. Se aplican con mayor autonomía gestora, con el objeto de subsanar determinados desequilibrios surgidos entre los diferentes aspectos del bienestar social.

Un ejemplo revelador del empuje integrador de las TIC como reductoras de asimetrías socioterritoriales está en la heterogénea articulación de acciones contra la exclusión digital en forma de servicio efectivo. Existen diferencias interregionales, y también a diferentes escalas político-administrativas, en el tratamiento de la cuestión, desde los criterios de diseño e implementación de 
estructuras en red (puntos de acceso a Internet, aulas TIC...), hasta la propia definición del tipo de cobertura a prestar, en detrimento de una suficiencia en la equidad y en los efectos distributivos de esta política, dependiendo de las condiciones de partida del fenómeno, de los propios procesos de producción y asignación de acceso y aprovechamiento TIC, o de los resultados producidos (Díaz, 2002). Por el momento, no se aprecia unanimidad entre los actores sociales clave acerca del modelo de estrategia operativa global más adecuado, considerando los principios y compromisos adoptados en la Cumbre Mundial sobre la Sociedad de la Información (2003-2005), la propia implementación de los mismos a través de estrategias consensuadas a escala internacional, -como la Agenda Digital Europea-, y la revisión de los avances de la Cumbre, prevista para 2015 (UIT, 2005).

\section{DesarRollo de la RED GUADALINFo en ANDALucía}

La evolución histórica de la experiencia Guadalinfo en Andalucía está vinculada a diversos hitos que han conformado aspectos tan destacados como su significado, su organización o su funcionamiento, sin cuya consideración sería difícilmente comprensible la actual realidad de impulso público y su consolidación como estrategia colectiva, cercana a la ciudadanía y basada en la colaboración en red entre sus agentes impulsores/dinamizadores y el conjunto de la sociedad beneficiaria.

De acuerdo con esta reflexión, es preciso comenzar por la destacada acción de ampliación por el territorio andaluz de la experiencia Guadalinfo, tras los óptimos resultados registrados con el Programa Regional de Acciones Innovadoras Guadalinfo 2002-2004, una prueba inicial de 26 telecentros dirigida a evaluar las posibilidades de una red regional de centros de acceso público a Internet. Los retos planteados por las nuevas tecnologías en el contexto de la sociedad de la información pusieron de manifiesto la necesidad de llevar a cabo algo más que un proyecto piloto. En consecuencia, se constató la oportunidad de una estrategia global para afrontar los cambios que ya se estaban observando en la sociedad, identificándose especialmente esta nueva forma de exclusión social basada, en esos momentos, en la incapacidad de acceso a las TIC y a internet. El escenario inicial del Programa coincide con la presencia de municipios poco poblados, situados principalmente en espacios rurales con débil desarrollo socioeconómico, déficit de acceso a Internet de banda ancha e insuficientes recursos públicos locales para hacer frente a la brecha digital.

Comienza así una etapa de desarrollo Guadalinfo que tiene como referentes de empuje la aprobación del Decreto 72/2003, de 18 de marzo, de Medidas de Impulso a la Sociedad del Conocimiento en Andalucía, y la posterior firma de un Convenio Marco 2004-2009 entre la Consejería de Innovación, Ciencia 
y Empresa (Junta de Andalucía) y las ocho Diputaciones Provinciales andaluzas para la progresiva implantación de centros de acceso público a Internet (2004-2006) en los 637 municipios andaluces con población inferior a 10.000 habitantes ( $82 \%$ del total de municipios andaluces) y su funcionamiento hasta 2009. Dicho compromiso general sería implementado a través de convenios específicos suscritos en cada provincia por las respectivas Diputaciones y los ayuntamientos de los citados municipios.

A raíz de la firma de estos convenios específicos se produce la gradual implantación de centros, destinados a prestar servicio a un conjunto de población cercano a los dos millones de habitantes ( $23 \%$ de la población andaluza), garantizando la cobertura de esta red a los municipios beneficiarios, cuyas prestaciones más importantes son el acceso a las TIC, la alfabetización digital básica o el uso de servicios y la adquisición de conocimientos por medio de Internet.

Un año después es aprobada la Orden de 20 de julio de 2007, reguladora de la concesión de incentivos para la apertura y explotación de centros de acceso público a Internet, dirigida a entidades asociativas con actuación en Zonas en Riesgo de Exclusión Social de municipios con población superior a 20.000 habitantes y a la habilitación de puntos de acceso público a Internet para Comunidades Andaluzas en el Exterior (CAE), ubicadas fuera de Andalucía, alcanzándose 26 centros de acceso público a Internet en barriadas desfavorecidas y un total de 17 centros en dichas CAE.

Si bien, es contemplado para los centros Guadalinfo un periodo operativo de seis años (2004-2009), se produce un cambio significativo en 2008, con la encomienda de gestión, optimización y sostenimiento de la red Guadalinfo al Consorcio Fernando de los Ríos ${ }^{2}$. Esta entidad pública instrumental es conformada por la Consejería de Innovación, Ciencia y Empresa (Junta de Andalucía) y las ocho Diputaciones Provinciales andaluzas para el impulso de la sociedad de la información, en coincidencia con importantes cambios o desafíos relativos a la propia filosofía de la política correctora de la brecha digital. Suscita un especial interés la ampliación de la red de equipamientos a municipios con población inferior a 20.000 habitantes, la consolidación de la experiencia iniciada en barriadas desfavorecidas o con mayor riesgo de exclusión social y el inicio de un nuevo modelo de atención ciudadana basado en la interacción ciudadana y el fomento de iniciativas innovadoras para la red.

Entre 2008 y 2010 la red alcanza los 693 centros, tras la incorporación de 56 nuevos equipamientos al amparo de la Orden de 7 de octubre de 2008 (Consejería

2. Resolución de 9 de julio de 2007, de la Dirección General de Administración Local, por la que se acordó la publicación de los estatutos modificados del Consorcio para el Desarrollo de Políticas en materia de Sociedad de la Información y el Conocimiento y posterior Resolución 13 de mayo de 2008, de la Dirección General de Administración Local, que rige la organización y funcionamiento de la entidad. 
de Innovación, Ciencia y Empresa), reguladora del programa de incentivos para la implantación y sostenimiento de la red de centros de acceso público a internet en municipios andaluces con más de 10.000 habitantes y menos de 20.000 habitantes. Esta realidad es completada con la consolidación y/o apertura de nuevos centros en barriadas desfavorecidas de municipios con población superior a 20.000 habitantes, llegándose a disponer de 42 equipamientos de esta categoría en 2009, aunque los puntos de acceso públicos a Internet desplegados en las CAE bajan a 16 unidades en 2010 .

Además de la impronta territorial de la realidad Guadalinfo en Andalucía, ha destacado la modernización de sus equipamientos y de sus prestaciones, como el impulso de nuevos mecanismos de formación en línea, la dinamización de su portal digital www.guadalinfo.es, -basado en los atributos de una red social-, o la promoción de la iniciativa ciudadana vía comunidades virtuales y la difusión de ideas innovadoras entre sus usuarios.

Este escenario de cambio es completado mediante el Plan Estratégico Guadalinfo 2009-2012, impulsado por la Comisión Ejecutiva del Consorcio, que consolida y sobrepasa esta etapa modernizadora para la red integradora, justificándose el ajuste producido en su funcionamiento a la propia evolución de las necesidades y oportunidades de la población andaluza. El Plan Estratégico amplía así el horizonte de aplicación de la política Guadalinfo hasta 2012, a modo de compromiso en la consolidación de los resultados conseguidos, desde la fuerza cooperadora y gestora del Consorcio. Para ello, fija como meta ser y potenciar el entorno digital en el que la sociedad andaluza progrese cultural, económica y socialmente, todo ello guiado por los principios de innovación, correspondencia, participación, excelencia, igualdad, sostenibilidad, cooperación y diversidad.

La dificultad para impulsar una nueva figura integradora ante el fin de la vigencia del Plan Estratégico, en pleno contexto de crisis económica, fue compensada mediante un marco financiero (2011-2013) que supone la ampliación del periodo de funcionamiento para los centros ubicados en municipios de hasta 20.000 habitantes $^{3}$. En este caso, se establece una triple distinción de estos centros de iniciativa pública municipal, con un Grupo A (189 municipios de menos de 1.000 habitantes), un Grupo B (448 municipios de entre $1.001 \mathrm{y}$ 10.000 habitantes) y un Grupo C (56 municipios de entre 10.001 y 20.000 habitantes). Mientras que las otras categorías de centros en barriadas desfavorecidas y comunidades andaluzas en el exterior pudieron prorrogar su funcionamiento

3. Orden de 15 de diciembre de 2010, reguladora de subvenciones a Corporaciones Locales de Andalucía para proyectos de mantenimiento de la red Guadalinfo (periodo 2011-2013). 


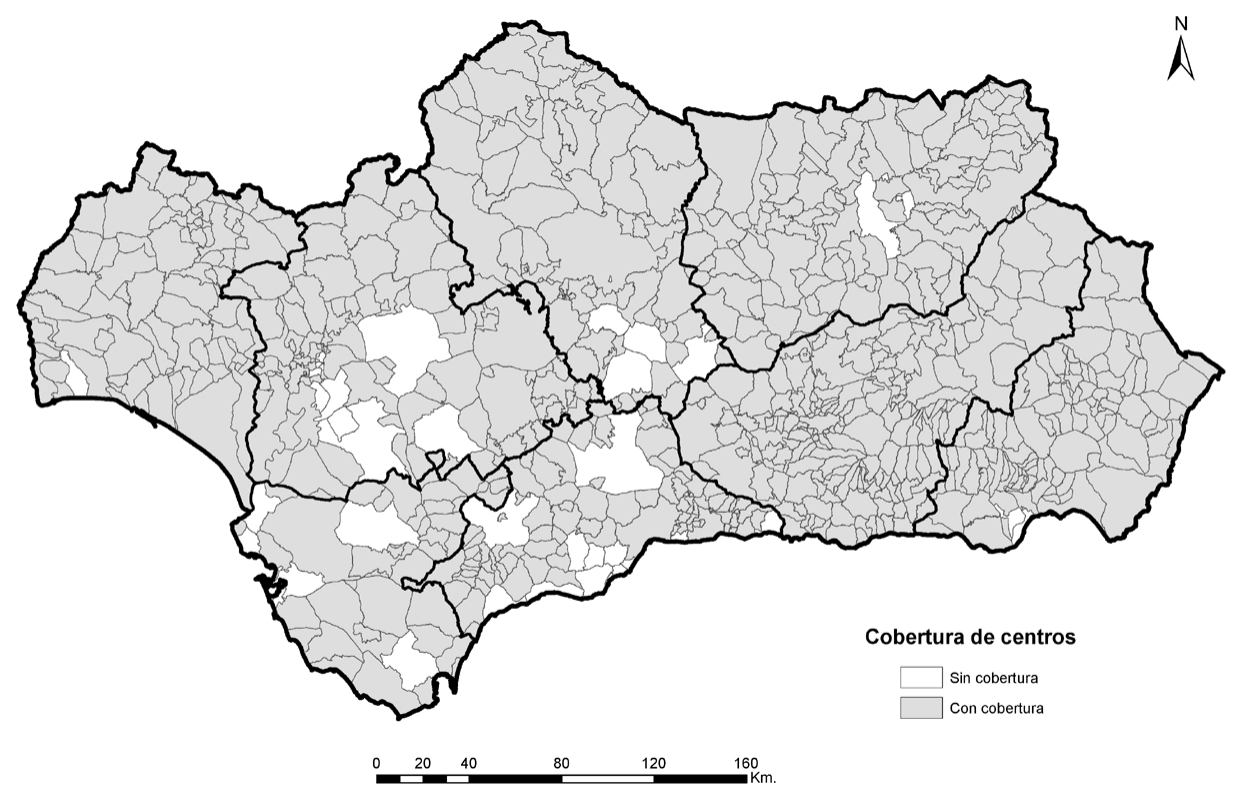

Mapa 1. Cobertura de la red Guadalinfo en Andalucía. Fuente: Elaboración propia, a partir de Guadalinfo.

anualmente ${ }^{4}$, fijando esta prestación en 64 barriadas y en 26 puntos de acceso en el exterior de Andalucía. De este modo, la red alcanza un número sin precedentes de equipamientos ( 783 centros) en su acción contra la brecha digital en la Comunidad.

Los diversos cambios producidos en el marco socioterritorial del proyecto Guadalinfo han resultado en gran medida fruto de un modelo de implantación e implementación vinculado al contexto estratégico externo, destacando sobremanera la incidencia directa de las políticas de inclusión digital y promoción de la sociedad de la información en el seno de la Unión Europea, en concreto destacando sus experiencias eEurope (2002 y 2005), iEurope 2010 y la posterior Agenda Digital 2020.

Si el principio de esta experiencia inclusiva en Andalucía coincidió con la extensión de un doble servicio de acceso gratuito a Internet y de alfabetización digital mediante una red experimental de centros ubicados en ámbitos locales con escasa población (Programa Regional de Acciones Innovadoras Guadalinfo 2002-2004), -como oportunidad compensadora de carencias

4. Resolución de 28 de febrero de 2011, de la Secretaría de Estado de Telecomunicaciones y para la Sociedad de la Información, prorrogando colaboración con la Junta de Andalucía. 
propias de municipios peor posicionados desde el punto de vista socioeconómico-, a medida que avanzó en su funcionamiento, extendiéndose al conjunto de municipios andaluces, esta política fue reinterpretada en su rol de medio de integración sociodigital, corrigiendo la inequidad socioterritorial y dinamizando a la ciudadanía en el uso e innovación de contenidos digitales, en coherencia con las directrices europeas de intervención, con la filosofía que encarna la red Telecentre Europe Community Networking y con integración en el Plan de Acción Europeo en Competencias Digitales.

\section{IMPACTOS MÁS DESTACADOS DE LA RED}

La obtención de un conocimiento más completo y detallado de aquellos impactos que la red de centros Guadalinfo ha tenido en el ámbito de la integración sociodigital en Andalucía, representa para este trabajo de investigación su pilar de análisis más destacado. Esta labor ha contado como soporte estadístico la información obtenida mediante 1.000 encuestas dirigidas a la población anda-

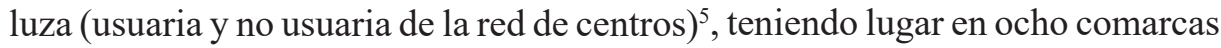
seleccionadas, distribuidas equitativamente por las provincias de la Comunidad: Campo de Tabernas (Almería), Costa Noroeste de Cádiz, Medio Guadalquivir (Córdoba), Guadix (Granada), Andévalo Occidental (Huelva), Jaén (Zona Metropolitana), Málaga (Zona Metropolitana) y Sierra Norte de Sevilla.

A partir de las opiniones emitidas se ha podido avanzar en la comprensión de la inclusión sociodigital en esta Comunidad Autónoma, comprobando su impronta y aquellas posibles vías de optimización de la acción reductora de la brecha digital. En concreto, se han examinado las mejoras globales más representativas, las temáticas con mayor atractivo para los 174 beneficiarios de centros de la red Guadalinfo ${ }^{6}$, la percepción del aprovechamiento ciudadano de los servicios ofrecidos, las opciones de futuro percibidas para los centros de integración sociodigital y las expectativas de la red en su formato de servicio a la ciudadanía.

\section{Referentes iniciales}

Se ha realizado una caracterización general de la exclusión digital de acuerdo con el sexo, la edad, la situación sociolaboral y el nivel de estudios de los

5. El tamaño de la muestra se ha calculado mediante la fórmula $4 \mathrm{~N} \mathrm{x} \mathrm{V} / \mathrm{NK}^{2}+4 \mathrm{~V}^{\prime}$, considerando una población objetivo (N) con 15 o más años de edad ( 997.060 personas), una cuasivarianza (V') basada en la variable Número de personas que acuden a los centros de la red Guadalinfo $\left(\mathrm{V}^{\prime}=250\right)$ y un error muestral $(\mathrm{K})$ del $10 \%$.

6. Este estudio ha reflejado un porcentaje de usuarios de centros de la Red Guadalinfo del 17,4\% sobre el total de la población encuestada. Así, las respuestas obtenidas sobre estos niveles de impacto y expectativas de futuro se corresponden únicamente con dichos usuarios. 
encuestados, así como con la disponibilidad de equipamiento informático y de conexión a Internet por parte de la población en los hogares, determinando de este modo sus circunstancias y el perfil de las personas colaboradoras.

La exclusión sociodigital asociada al sexo ha sido una realidad entre la ciudadanía encuestada, demostrándose un predominio de las mujeres en los niveles inferiores y medio de acceso y manejo digital, donde se ha concentrado el 92,2\% de encuestados. Por el contrario, es en los niveles alto y muy alto de inclusión digital donde son mayoría los hombres. De este modo, se ha podido confirma la inequidad de género en la sociedad de la información que diversos estudios han venido alertando.

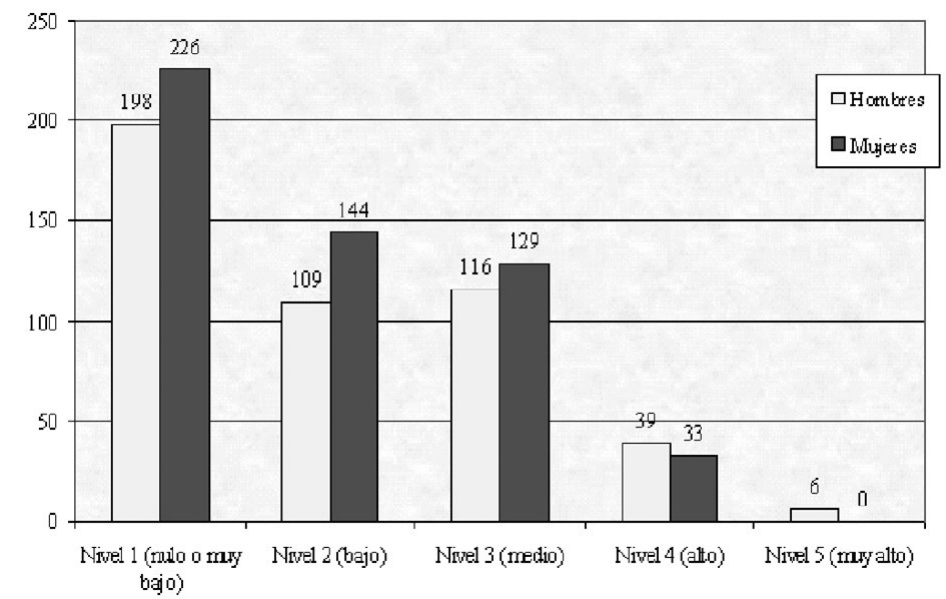

Gráfico 1. Distribución general del acceso y manejo TIC e Internet según sexo. Fuente: Elaboración propia.

La diferenciación sociodigital en función de la edad resulta altamente significativa, de acuerdo con los resultados alcanzados. Los grupos de encuestados con edad superior a los 45 años se han concentrado en los niveles más bajos de acceso y manejo de las TIC e Internet. En contraposición, el grupo de personas menores de 25 años ha mostrado un mayor acceso y conocimiento de las nuevas tecnologías y de Internet, predominando en los niveles medio y alto al respecto. Así mismo, se ha registrado un número anecdótico de personas con capacidades muy altas, todas ellas menores de 65 años. 


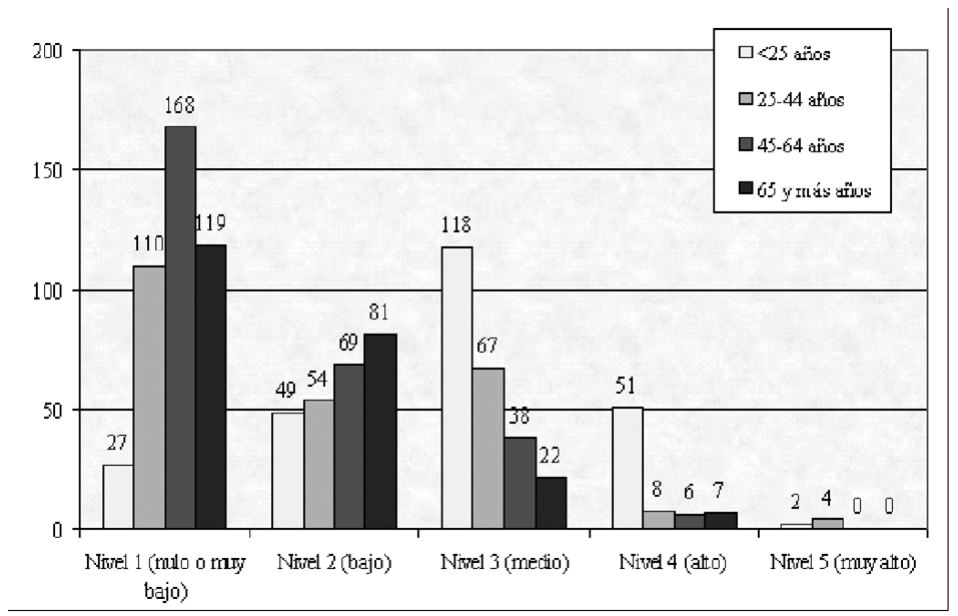

Gráfico 2. Niveles de acceso y manejo TIC e Internet según edad. Fuente: Elaboración propia.

Atendiendo al cotejo de la situación laboral de las personas encuestadas con su capacidad de acceso y manejo de las TIC e Internet, el reparto obtenido puede ser interpretado bajo diversos apartados de notoriedad. Uno de éstos es el caso de la población ocupada, cuyo carácter dominante en un nivel nulo o muy bajo de acceso y manejo TIC ha evidenciado una carencia significativa en su capacitación de cara a determinados requerimientos asociados a sus respectivos cometidos laborales, situación apreciada también en los niveles bajo y medio.

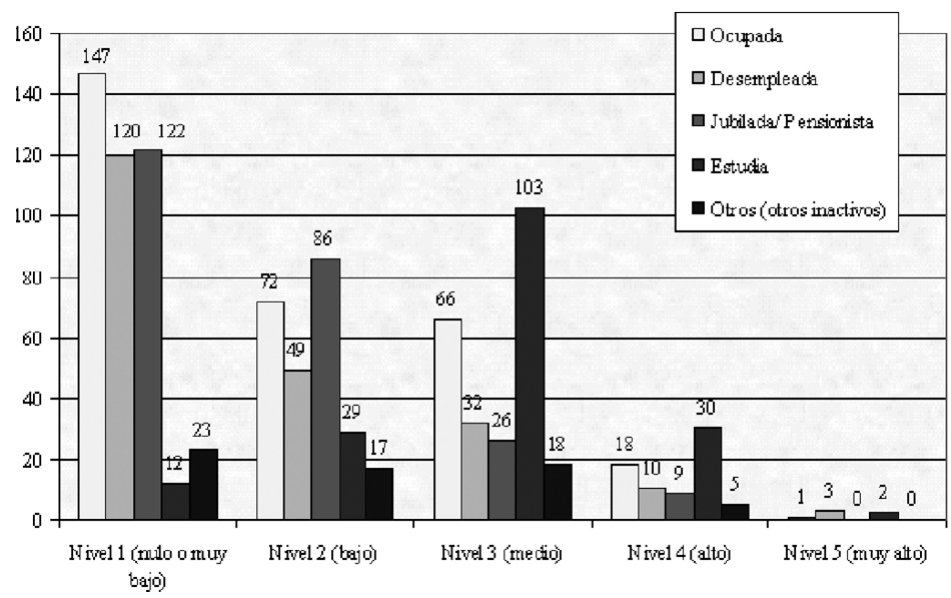

Gráfico 3. Niveles de acceso y manejo TIC e Internet según situación laboral. Fuente: Elaboración propia. 
La población desempleada ha mostrado, asimismo, un acceso y capacitación similar al grupo anterior, pese a las condiciones de cambio y las exigencias de adaptación a un mercado de trabajo cada día más exigente, en el que las TIC tienden a generalizar su uso. Al mismo tiempo, el sector de jubilados/pensionistas se ha caracterizado por una concentración de sus efectivos en los niveles inferiores de acceso y manejo TIC, dificultando su acercamiento a determinados servicios de interés, como sanidad y servicios sociales, ocio o acceso general a la información, sujetos a nuevas fórmulas de interacción en línea.

Por último, cabe destacar el elevado número de estudiantes con niveles registrados de acceso y manejo medio y alto. Dichos resultados evidencian una ruptura importante en la tónica general de población examinada y su mayor grado de inmersión en la sociedad de la información.

El nivel de estudios de la población guarda una estrecha relación con su capacitación o forma de desenvolverse en materias tan diversas como la cultura, el empleo, las relaciones sociales o el acceso y aprovechamiento de los avances tecnológicos. Las encuestas realizadas han permitido mostrar interesantes vínculos entre el grado de instrucción ciudadana y su capacidad de acceso y manejo de las TIC e Internet.

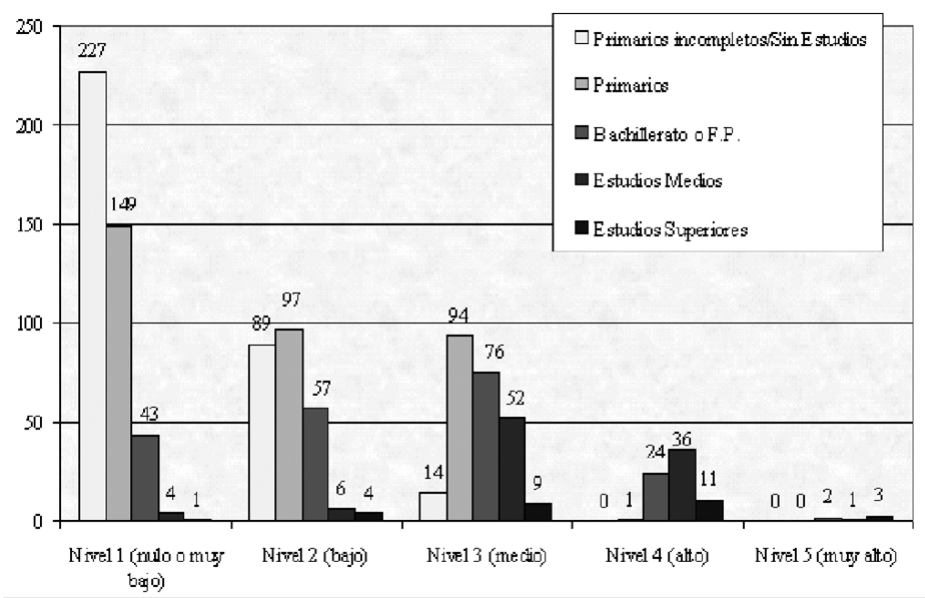

Gráfico 4. Niveles de acceso y manejo TIC e Internet según capacitación personal. Fuente: Elaboración propia.

La mayor incapacidad de acceso y manejo digital ha coincidido con los grupos de población menos formada, aunque hay que matizar dicha relación considerando la edad de los encuestados. Así, determinadas personas con estudios de Bachillerato/Formación Profesional, medios o superiores, con más de 65 años, 
han manifestado un reducido acceso y manejo de medios digitales, por pertenecer a generaciones temporalmente alejadas de las nuevas tecnologías de la información y la comunicación.

Es en el nivel intermedio de acceso y manejo digital donde se ha apreciado un mayor equilibrio de todos los grupos formativos examinados, mientras que los niveles de acceso y manejo TIC más elevados concentran población con titulaciones que superan los estudios primarios.

Tras el análisis de la formación ciudadana se puede confirmar la existencia de bolsas de exclusión sociodigital entre aquellas personas con niveles de estudios nulos o más elementales, produciéndose una situación inversa en los casos de aquellos entrevistados cuyos grados de instrucción se sitúan en los escalafones más altos.

Junto a los marcadores de inequidad observados, ha resultado fundamental para el conocimiento de la situación de partida la opinión de los encuestados acerca de la disponibilidad de equipos informáticos y de conexión a Internet, que avale la necesidad de acceder a las prestaciones de la red Guadalinfo. Comenzando por la disponibilidad de equipo informático (ordenador personal) propio en el hogar, se han obtenido 551 respuestas favorables, frente a 449 desfavorables.

Aunque se aprecia un cierto equilibrio entre personas con y sin disponibilidad de equipos informáticos, se ha evidenciado la existencia de determinados tipos de carencias obstaculizadoras de la inclusión sociodigital. Ha resultado muy reveladora la justificación por parte de las personas encuestadas a dicha carestía, altamente significativa en los casos de insuficiencia de medios económicos y de desconocimiento sobre su uso (sumando ambas el 35,7\% de las respuestas emitidas). Por otra parte, la falta de tiempo para dedicar al uso de las TIC ha revelado una doble adversidad, tanto física como temporal para sus afectados.

La carencia de conexión a Internet en el hogar ha sido también representativa entre la población encuestada, aunque su desproporción ha sido mayor que en el caso anterior, entre quienes poseen conexión a la red (424 individuos) y aquellos que han manifestado su carencia (576 casos). Estos resultados han demostrado la existencia de personas con equipo informático en casa, sin llegar por ello a estar conectadas a internet. La carencia de este servicio ha venido justificada en su mayor parte por la falta de conocimiento para disfrutarlo, seguida de una insuficiencia de recursos económicos para disponer del mismo. A más distancia se ha reconocido la carencia de tiempo para su aprovechamiento y el desinterés hacia sus ventajas y prestaciones. 


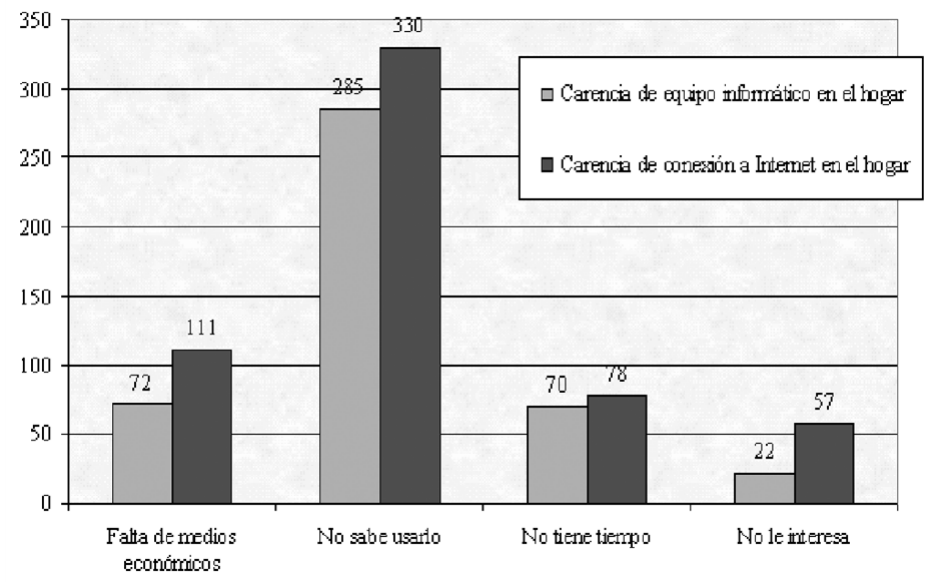

Gráfico 5. Causas generales de la carencia TIC y de acceso a Internet en el hogar. Fuente: Elaboración propia.

Finalmente, ha resultado altamente significativa la diferencia existente entre declarantes de unos niveles muy bajos de acceso y conocimiento en el manejo de las TIC e Internet (677 encuestados) y una necesidad importante de mejorar dicha situación (681 casos), respecto de los 174 usuarios reales de los centros encuestados, demostrando el enorme potencial de la red para captar nuevos beneficiarios de su acción capacitadora contra la brecha digital.

\section{Impactos y expectativas para la red}

Los impactos globales que la red Guadalinfo ha provocado entre las personas usuarias de los centros analizados han quedado agrupados en cuatro modalidades, con la idea de comprobar el reconocimiento recibido centrado en las posibilidades de acceso a recursos TIC e Internet, la iniciación en el manejo de las TIC y sus aplicativos básicos (alfabetización digital), el progreso en el aprovechamiento de estos recursos electrónicos y la dinamización en comportamientos innovadores (interrelación con comunidades de usuarios, lanzamiento de ideas y aplicaciones o propuestas de iniciativas para la red). 


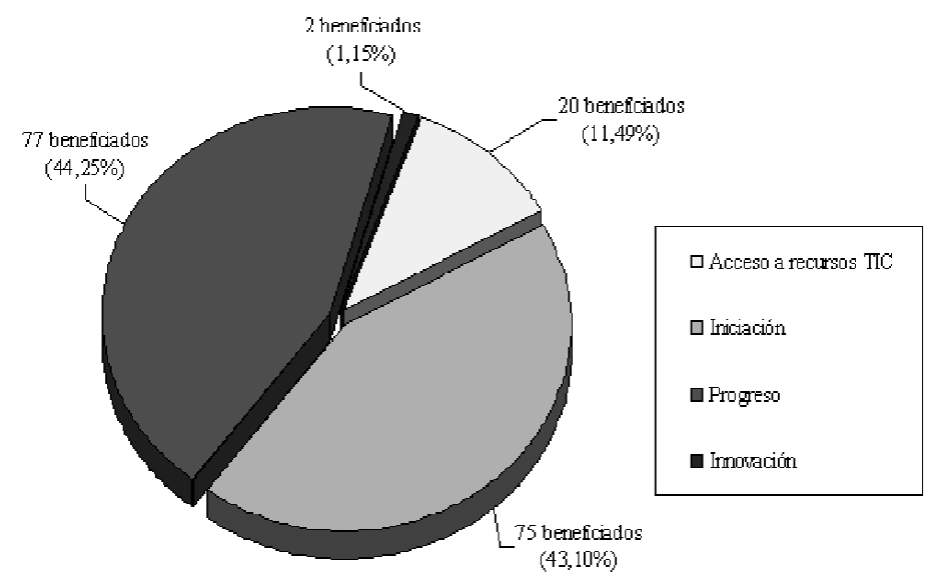

Gráfico 6. Impactos globales reconocidos por los usuarios encuestados. Fuente: Elaboración propia.

Como puede apreciarse, ha existido un reparto casi idéntico entre los 174 beneficiarios de centros encuestados que señalan la iniciación y el progreso en el manejo y aprovechamiento TIC y de Internet, mientras que el acceso a los recursos tecnológicos ofrecidos por los centros resulta ser el tercer beneficio mejor valorado entre estos usuarios, aunque vinculado sólo a una minoría de ellos. Por último, el efecto beneficioso de la innovación es considerado como el principal impacto para un número poco representativo de personas consultadas, resultando ser una opción escasamente visualizada en los centros y que implica a un reducido número de usuarios con inquietudes creativas y con un manifiesto deseo de implicarse en la divulgación de experiencias, conocimientos o ideas exitosas, también conocidos por los gestores de la red como usuarios motores.

Junto a los impactos globales, han sido objeto de escrutinio los servicios y actividades de inclusión sociodigital generados en los centros, solicitándose a los usuarios encuestados la identificación de las tres temáticas concretas más atractivas desde el punto de vista de su beneficio personal. De las 522 respuestas emitidas destacaron por su número de nominaciones la búsqueda de información (25,1\% de casos), la comunicación y relaciones sociales (20,1\%), la formación y perfeccionamiento en el manejo y aprovechamiento TIC e Internet $(18,8 \%)$ y la alfabetización digital $(15,9 \%)$.

De este modo, ha sido posible advertir una diversificación muy destacada en el aprovechamiento de los diferentes servicios ofrecidos por los centros de la red Guadalinfo, aunque centrada especialmente en la cualificación personal en 
el uso de los recursos electrónicos disponibles y el manejo de sus prestaciones más básicas, mostrando aún desafección hacia aspectos más avanzados de la sociedad de la información, como el comercio electrónico o la interacción con diversas instituciones de interés, incluida la e-Administración).
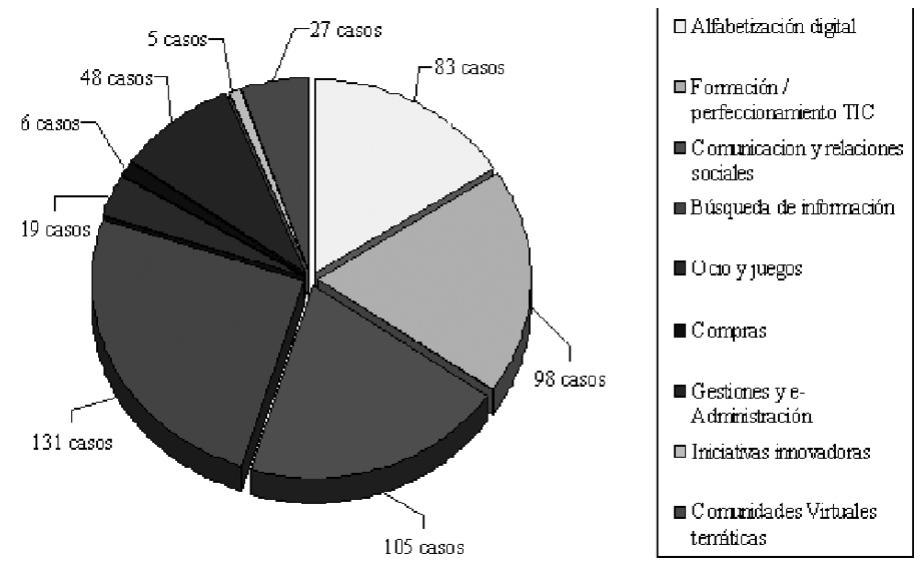

Gráfico 7. Distribución de las temáticas de centro más atractivas. Fuente: Elaboración propia.

El reconocimiento por parte de los usuarios del impacto que la red de centros de acceso público a Internet ha tenido sobre el conjunto de la población andaluza, en forma de aprovechamiento de su carta de servicios, recursos y prestaciones, ha sido estudiado también, intentando así verificar la actitud ciudadana hacia las ventajas y oportunidades ofrecidas por la red Guadalinfo en su pretendida labor de inclusión sociodigital.

Preguntados por el nivel de disfrute ciudadano de los centros, se ha producido una fuerte concentración de opiniones en torno a los estratos bajo y medio de interés (sumando el $81,6 \%$ de las respuestas), que en su mayoría se han identificado con una insuficiente o defectuosa labor divulgativa por parte de sus gestores. De este modo, sería posible aumentar el número de usuarios encarando esta deficiencia e indagando en torno a las circunstancias y expectativas de la población desvinculada de los centros. 


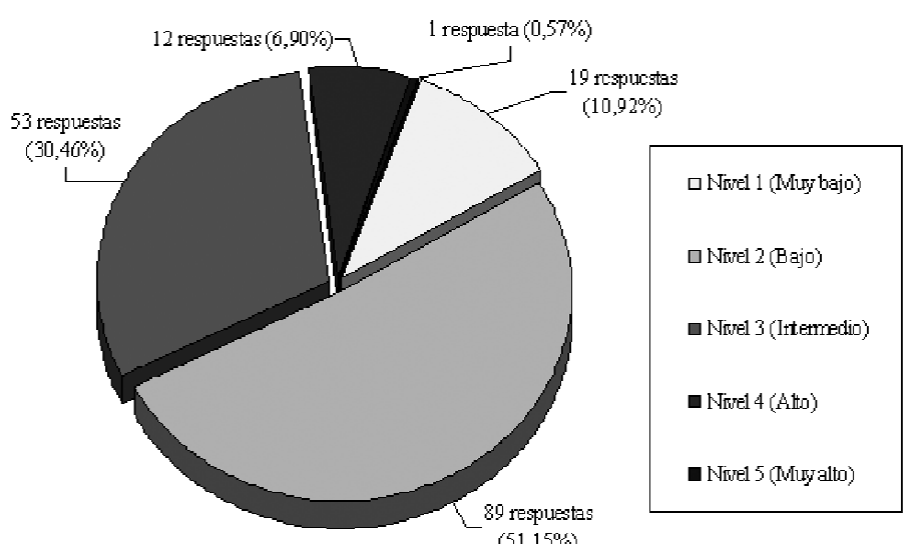

$(51,15 \%)$

Gráfico 8. Distribución del nivel de aprovechamiento percibido de los centros. Fuente: Elaboración propia.

El examen de las potencialidades de la red ha posibilitado el conocimiento de aquellas mejoras o novedades de sus servicios y de sus expectativas globales ante la brecha digital. Comenzando por los avances concretos reconocidos para estos centros (sus respuestas emitidas), se han posicionado positivamente el contacto directo con especialistas en tecnologías y temáticas digitales de elevado interés para los usuarios ( $27,9 \%$ de respuestas), seguido de medios técnicos y aplicativos actualizados $(20,9 \%)$ y de la difusión de la innovación y resultados relacionados con la sociedad de la innovación $(18,8 \%)$.
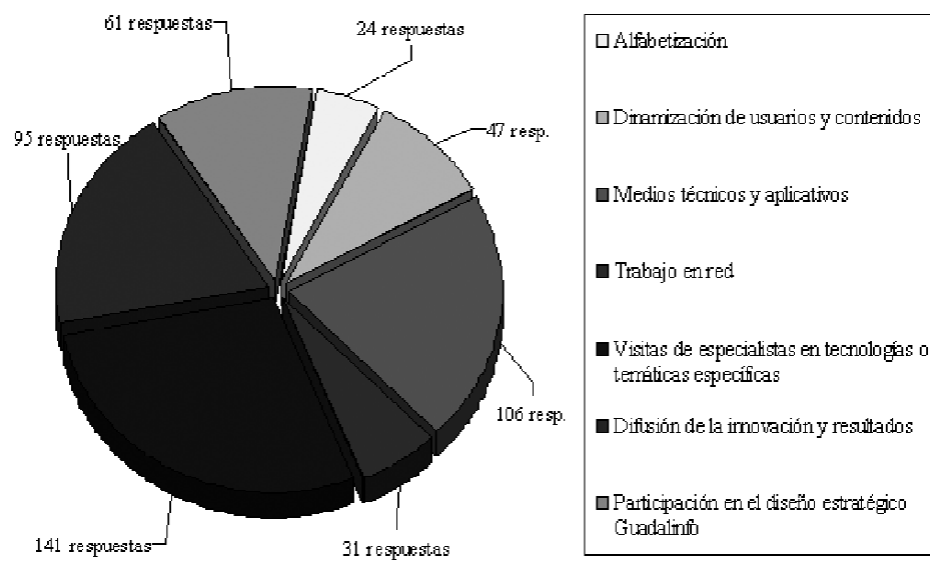

Gráfico 9. Mejoras percibidas para los centros de la red Guadalinfo. Fuente: Elaboración propia. 
La participación en el diseño estratégico de los servicios y mejoras en los centros Guadalinfo ha supuesto la cuarta aspiración ciudadana en importancia (12,1\%), argumentada por una preocupación abierta hacia la elección del plan de trabajo a implementar, considerando la capacidad de la red para dinamizar la participación ciudadana y la retroalimentación de su línea estratégica.

La segunda expectativa analizada se ha referido al cometido de la red Guadalinfo en su condición de servicio público de atención ciudadana, considerando dos perspectivas distintas aunque complementarias: interactuando con otros servicios tradicionales de inclusión social, vinculados a políticas de educación, formación o bienestar social, en general, e incidiendo específicamente en el acceso y la cualificación para el uso y aprovechamiento de las TIC e Internet.

Tras pedir a los usuarios encuestados su opinión acerca de estas posibilidades, las respuestas emitidas han evidenciado una importante división sobre la futura orientación de los centros. Aunque ha sido sensiblemente mayoritaria la opción integradora, que pretende reforzar la complementariedad de la red con otros servicios institucionales asociados al bienestar social y a la cualificación ciudadana (47,13\% de apoyos), sería injusto desestimar la visión de un papel exclusivo en la inclusión digital, al existir una diferencia de sólo 6,9 puntos porcentuales entre ambas modalidades.

Conviene considerar también el $12,64 \%$ de usuarios indecisos, cuya indefinición viene dada porque no ven alternativas claras al actual modelo de centros, o bien, son incapaces de decantarse por una de las opciones planteadas, al considerarlas igualmente válidas.

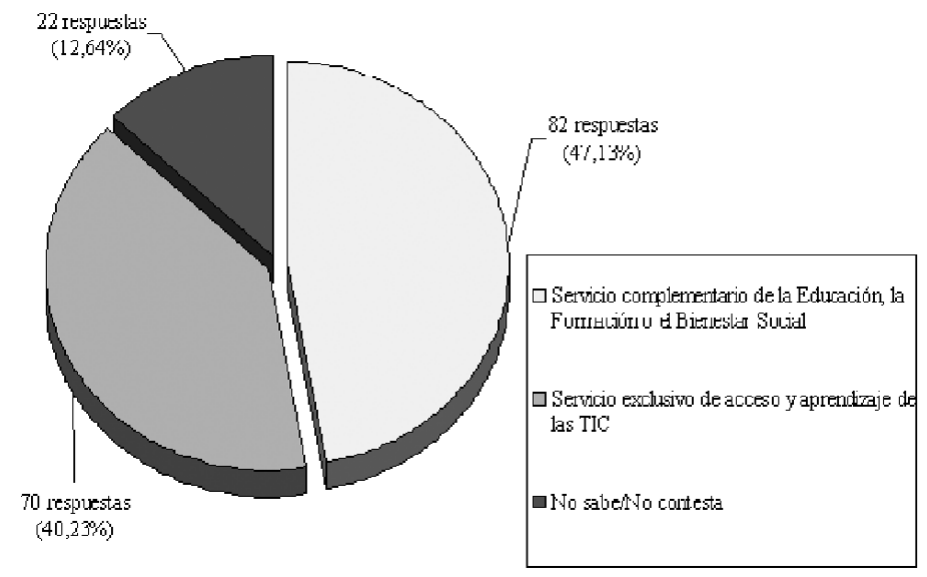

Gráfico 10. Expectativas generales de los usuarios sobre el servicio de la red. Fuente: Elaboración propia. 
Estas circunstancias hacen pensar en la necesidad de acometer un profundo debate social e institucional acerca de la política de inclusión sociodigital en Andalucía, mejorando así su gobernanza y apuntando con mayor eficacia a las necesidades y expectativas surgidas en estos años de ejercicio.

\section{CONCLUSIONES}

Las conclusiones más destacadas del trabajo quedan agrupadas en torno a tres grandes bloques de exposición relacionados con la visión y posicionamiento de la Administración Andaluza responsable de la política de inmersión en la sociedad de la información, el proceso de extensión de la red de inclusión sociodigital que representa a la estrategia Guadalinfo, así como los impactos concretos de los centros de atención ciudadana que componen dicha red.

Por un lado, se ha podido constatar un modelo de política regional contra la brecha digital que confirma su visualización de la inequidad más allá de posicionamientos amparados en la simple carencia de medios tecnológicos y de acceso a los mismos por parte de la población andaluza, o de falta de cualificación digital. Más bien, se ha podido apreciar una forma de acometer dicha inequidad basada en la intervención integral, no exenta de dificultades o de carencias en la expresión territorial de su red de centros, ni del debido consenso en la movilización de recursos de acuerdo con una visión global del servicio que se ofrece a la ciudadanía.

Por otra parte, se ha logrado identificar el complejo proceso creativo de la actual red de inclusión Guadalinfo, desde unos comienzos inciertos en 2002, en el intento de compensar destacados vacíos digitales relacionados con el carácter rural y de aislamiento geográfico una parte importante del territorio andaluz, con manifiestas carencias en materia de infraestructuras digitales e insuficientes recursos económicos de las administraciones locales para afrontar acciones de acercamiento de las TIC e Internet a la ciudadanía, todo ello al amparo de los impulsos europeos del momento (iniciativa eEurope 2002) que actúan como contexto estratégico de referencia.

El crecimiento de la red y su generalización en casi todo el territorio de la Comunidad Autónoma ha tenido como denominador común la progresividad, en forma de fases o impulsos generadores que han dado crecimiento a dicha red y conformado un modelo de gestión basado en la cooperación entre diversas instituciones, desde el Consorcio Fernando de los Ríos, compuesto por la Administración Autonómica y las Diputaciones Provinciales andaluzas, hasta un conjunto de 693 ayuntamientos, 64 entidades asociativas sin ánimo de lucro y 26 Comunidades Andaluzas situadas en el exterior de Andalucía. No obstante, cabría señalar la permanencia de importantes vacíos de cobertura, si se considera la ausencia de estos equipamientos en aquellas barriadas no señaladas 
como desfavorecidas de municipios o ciudades mayores de 20.000 habitantes, donde también se hace presente la brecha digital, como refleja el conjunto global de carencias y expectativas expresadas por la población encuestada.

La encuesta realizada ha permitido constatar un perfil de afectados por la brecha digital en Andalucía, con un predominio del sexo femenino y de aquellas personas con edades más avanzadas, los cuáles han reconocido unos niveles más precarios de acceso y manejo digital. De acuerdo con la situación sociolaboral de los encuestados, no ha sido posible establecer una diferencia clara, compartiendo dicha adversidad tanto personas ocupadas como desempleadas y jubiladas/pensionistas, lo que hace pensar en una gran heterogeneidad de casos, aunque en su mayoría vinculados a un grado de capacitación personal nulo o muy elemental (estudios primarios, ya sean completos o incompletos).

Junto a lo anterior, se ha podido apreciar una disponibilidad limitada de equipos informáticos y de conexión a Internet en los hogares, derivada principalmente de la falta de conocimiento acerca de su uso y por la insolvencia económica para disfrutarlos, todo ello acompañado de un reducido aprovechamiento del acceso y uso a los mismos por parte de la red de centros Guadalinfo (si un 68,1\% de los encuestados ha reconocido la necesidad de un nivel adecuado de acceso y capacitación digital, sólo un 17,4\% han resultado ser usuarios de esta red de integración).

En cuanto a los impactos y expectativas recogidos en este estudio, cabe destacar el predominio de las acciones formativas como beneficio más extendido, quedando en un segundo término el acceso a los medios TIC e Internet. Concretamente, se ha producido un importante impacto social en el acercamiento al manejo de los medios digitales y su aprovechamiento para las comunicaciones en las relaciones sociales y la búsqueda de información relevante. Como contrapunto a estos resultados, se ha podido confirmar el reconocimiento de un bajo aprovechamiento de los centros de la red Guadalinfo por parte de la población en general, algo ya observado al comprobar la discrepancia entre usuarios y personas con necesidades de inclusión sociodigital.

Las expectativas visualizadas demuestran la fortaleza de la red, cuyos usuarios demandan una constante actualización, especialización y difusión de sus recursos y prestaciones, incluida su implicación en la toma de decisiones de su estrategia de futuro. Sin embargo, no ha sido posible consensuar un modelo de servicio definitivo para esta red, al producirse una polarización de opiniones entre quienes perciben una clara complementariedad de la inclusión/dinamización digital con las políticas existentes de inclusión social (educación, cultura, bienestar social...) y un número similar de opiniones que reivindican su empleo exclusivo como medio de acceso y capacitación en el aprovechamiento de los medios digitales. 
Ante estas consideraciones expresadas se abre un amplio camino por recorrer en la investigación de esta política regional, comprobando los avances que se vayan produciendo en los próximos años, e igualmente importante resultará su comparación con los resultados obtenidos en otros ámbitos geográficos del entorno, contribuyendo así a compensar determinados vacíos en las estadísticas oficiales disponibles y a elevar el nivel de conocimiento de esta estrategia integradora de cara a su mejor gestión.

\section{BIBLIOGRAFÍA}

BAWDEN, D. (2002): «Revisión de los conceptos de alfabetización informacional y alfabetización digital», en Anales de Documentación, n. ${ }^{\circ}$ 5, pp. 361-408.

BrandtzæG, P. B., Heim, J. y Karahasanović, A. (2010): «Understanding the new digital divide- A typology of internet users in Europe», en International Journal of Human - Computer Studies, n. ${ }^{\circ} 69$ (3), pp. 123-138.

Camacho, K. (2005): La brecha digital. En Ambrosi, A., Peugeot, V. y Pimienta, D.: Palabras en juego: Enfoques multiculturales sobre la sociedad de la información. C \& F Éditions. Paris, pp. 61-69.

Castells, M. (2006): La sociedad red: una visión global. Alianza Ed. Madrid, 558 pp.

Comisión Europea (2010): Comunicación de la Comisión al Parlamento Europeo, al Consejo, al Comité Económico y Social Europeo y al Comité de las Regiones. «Una Agenda Digital para Europa». [COM (2010) 245 final/2]. 46 pp.

Consejería de Innovación, Ciencia y Empresa (2004): PRAI Guadalinfo: Una visión global. Junta de Andalucía. Sevilla, 70 pp.

Consejería de Innovación, Ciencia y Empresa (2006): Plan Andalucía Sociedad de la Información. Junta de Andalucía. Sevilla, $112 \mathrm{pp}$.

Consejería de Innovación, CiENCIA Y EMPRESA (2009): Tenemos un plan. Plan Estratégico Guadalinfo 2009-2012. Junta de Andalucía. Sevilla, 35 pp.

Chaparro, J. (2007): «La segregación digital en contexto», en Ar@cne. Revista electrónica de recursos en Internet sobre Geografía y Ciencias Sociales. Universidad de Barcelona, n. ${ }^{\circ} 95$.

DAVENPORT, T. H. (1997): Information Ecology: Mastering the Information \& Knowledge Environment, Oxford University Press. Oxford, 272 pp.

DíAz, J. M. (2001): «Evaluación de la equidad y los efectos distributivos de las políticas públicas», en Actas del VII Congreso Internacional sobre la Reforma del Estado y de la Administración Pública. CLAD. Lisboa, pp. 1-16.

DiMaggio, P., Hargittai, E., Celeste, C. y Shafer, S. (2004): Digital Inequality: From Unequal Access to Differentiated Use. En Neckerman, K. M. (Ed.): Social Inequality. Russell Sage Fundation. New Cork, pp. 549-566

Downing, J. (comp.) (2000): Radical media: Rebellious communications and social movements. Sage Publications. London, $427 \mathrm{pp}$.

GARcía, I. (2005): «Políticas para la inclusión social mediante tecnologías de la información y la comunicación», en Revista Electrónica Teoría de la Educación: Educación y Cultura en la Sociedad de la Información, n. ${ }^{\circ} 6$ (1). 
Guerra, M. y Jordán, V. (2010): Políticas Públicas de Sociedad de la Información en América Latina: ¿una misma visión? Naciones Unidas. Santiago de Chile, 26 pp.

Gutierrez A. (2007). LA ALFABETIZACIÓN MÚLTIPLE EN LA SOCIEDAD DE LA INFORMACión. En CASADO, R. (coord.): Claves de la Alfabetización digital. Fundación Telefónica. Barcelona, pp. 73-85.

Hernández, M. (Coord.) (2008): Exclusión social y desigualdad. Murcia: Ediciones de la Universidad de Murcia, 414 pp.

Johnston, B. y WeBbeR, S. (1999): «Information literacy as an academic discipline», en 3rd British-Nordic conference on Library and Information Studies, Swedish School of Library and Information Science, University of Boras, Sweden.

KaraKayA, R. (2012): «Digital Exclusion in Turkey: A Policy Perspective», en Government Information Quarterly, n. ${ }^{\circ} 29$ (4): pp. 589-596.

LóPEz, P. y SAMEK, T. (2009): «Inclusión Digital: Un nuevo derecho humano», en Educación y Biblioteca (OEI), n. ${ }^{\circ}$ 172, pp. 114-118.

López, J. A. y Sánchez, J. M. (2001): Ciencia, Tecnología, Sociedad y Cultura en el cambio de siglo. Biblioteca Nueva, S.L. Organización de Estados Iberoamericanos (OEI). Madrid, $365 \mathrm{pp}$.

Manzanares, J. (2007): Alfabetización digital y ciudadanía. En: Casado, R. (Dir.): Claves de la alfabetización digital. Fundación Telefónica. Madrid, pp. 229-234.

Marco, M. (2000): «Consideraciones en torno al concepto de exclusión social», en Revista Acciones e Investigaciones Sociales, n. ${ }^{\circ} 11$, pp. 9-21.

Mattelart, A. (2007): Historia de la sociedad de la información. Paidós. Barcelona, $203 \mathrm{pp}$.

Norris, P. (2001): Digital divide? Civic engagement, information poverty and the Internet worldwide. Cambridge University Press. Cambridge, 303 pp.

OCDE (2001): Understanding the digital divide. OCDE. París, 33 pp.

SAmpedro, L. L. (2013): Economía Humanista. Algo más que cifras. Debolsillo. Barcelona, $429 \mathrm{pp}$.

Serrano, A. y Martínez, E. (2003) La brecha digital. Mitos y realidades. Departamento Editorial de la Universidad Autónoma de Baja California. Baja California, 133 pp.

Servon, L.; Pinkett, R. (2006): Estrechando la brecha digital: potencial y límites del movimiento de tecnología comunitaria norteamericano. En: Castells, Manuel (2006): La sociedad red: una visión global. Alianza Ed. Madrid, pp. 390-412.

SiLVER, A. (2009): «Aproximación europea a la educación en medios: avanzando hacia una sociedad del conocimiento inclusiva», en Comunicar, n. ${ }^{\circ} 32$, pp. 19-20

TARroja, À. y CAMAgni, R. (2006): Una nueva cultura del territorio. Criterios sociales y ambientales en las políticas y el gobierno del territorio. Diputación de Barcelona. Barcelona, $750 \mathrm{pp}$.

UIT (2005): Cumbre Mundial sobre la Sociedad de la Información. Documentos Finales. Ginebra 2003 - Túnez 2005. Unión Internacional de Telecomunicaciones. Ginebra, $112 \mathrm{pp}$.

VAn Disk, J. (2005): The Deepening Divide: Inequality in the Information Society. Sage Publications, INC. California, 248 pp. 